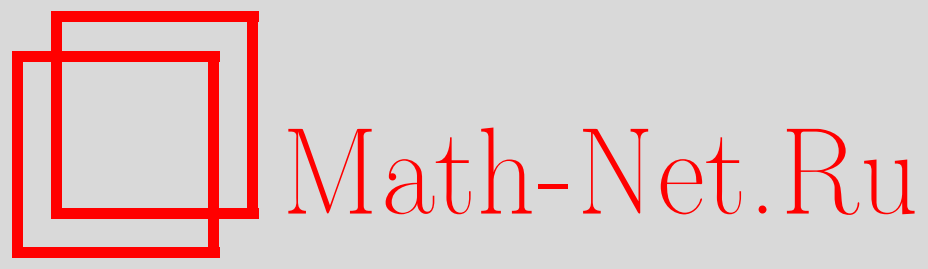

В. Л. Гейнц, В. В. Филиппов, О выпуклых оболочках компактных множеств вероятностных мер со счетными носителями, Функи. анализ и его прил., 2011, том 45, выпуск 1, 83-88

DOI: https://doi.org/10.4213/faa3000

Использование Общероссийского математического портала MathNet.Ru подразумевает, что вы прочитали и согласны с пользовательским соглашением http://www . mathnet.ru/rus/agreement

Параметры загрузки:

IP : 52.6 .47 .48

26 апреля 2023 г., 17:56:34

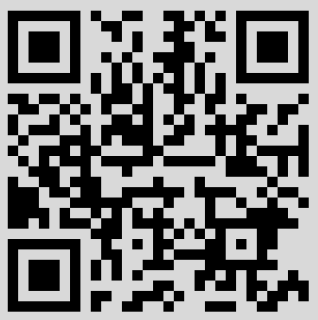


Банахово пространство $X$ имеет котип $q \geqslant 2$, если существует константа $K>0$, такая, что для произвольных векторов $x_{1}, \ldots, x_{n}$ из $X$

$$
\left(\sum_{k=1}^{n}\left\|x_{k}\right\|^{q}\right)^{1 / q} \leqslant K \int_{0}^{1}\left\|\sum_{k=1}^{n} r_{k}(t) x_{k}\right\| d t .
$$

Последнее определение будет иметь смысл и для $q=\infty$, если в левой части неравенства будет стоять $\max _{1 \leqslant k \leqslant n}\left\|x_{k}\right\|$. Говорят, что пространство $X$ имеет тривиальный тип (тривиальный котип), если оно не имеет типа, большего 1 (соответственно конечного котипа).

Как уже говорилось, пространство $\operatorname{Ces}_{p}(I)$ содержит копию пространства $L^{1}$. Поэтому из теорем 4 и 6 вытекает следующий результат.

Следствие 2. Если $1<p<\infty$, то пространство $\operatorname{Ces}_{p}(I)$ имеет тривиальный muп и котип $\max (p, 2)$. Пространство $\operatorname{Ces}_{\infty}(I)$ имеет тривиальнъй тип и тривиальный котип.

\title{
ЛитерАТУРА
}

[1] A. A. Jagers, Nieuw Arch. Wiskund. (3), 22 (1974), 113-124. [2] Б. И. Коренблюм, С. Г. Крейн, В. Я. Левин, Докл. АН СССР (н.с.), 62 (1948), 17-20. [3] W. Wnuk, Banach Lattices with Order Continuous Norms, Polish Scientific Publishers PWN, Warzaw, 1999. [4] J.-S. Shiue, Tamkang J. Math., 1 (1970), 91-95. [5] B. D. Hassard, D. A. Hussein, Tamkang J. Math., 4 (1973), 19-25. [6] P. W. Sy, W. Y. Zhang, P. Y. Lee, Glas. Mat. Ser. III, 22:1 (1987), 103-112. [7] Л. В. Канторович, Г. П. Акилов, Функииональный анализ, Наука, М., 1977. [8] С. Г. Крейн, Ю. И. Петунин, Е. М. Семенов, Интерполяиия линейных операторов, Наука, М., 1978. [9] J. Lindenstrauss, L. Tzafriri, Classical Banach Spaces, II. Function Spaces, Springer-Verlag, Berlin-New York, 1979. [10] G. Bennett, Factorizing the Classical Inequalities, Mem. Amer. Math. Soc., vol. 120, Amer. Math. Soc., Providence, RI, 1996. [11] W. A. J. Luxemburg, A. C. Zaanen, Math. Ann., 162 (1965/1966), 337-350. [12] K. Tandori, Publ. Math. Debrecen., 3 (1954), 263-268. [13] S. V. Astashkin, L. Maligranda, Proc. Amer. Math. Soc., 136:12 (2008), 4289-4294. [14] F. Albiac, N. J. Kalton, Topics in Banach Space Theory, Graduate Texts in Math., vol. 233, Springer-Verlag, New York, 2006. [15] M. I. Kadec, A. Pełczyński, Studia Math., 21 (1962), 161-176.

Самарский госуниверситет

Поступило в редакцию e-mail: astashkn@ssu.samara.ru 6 марта 2009 г.

Технологический университет Лулео, Швеция e-mail: lech@sm.luth.se

УДК 515.12

\section{О выпуклых оболочках компактных множеств вероятностных мер со счетными носителями}

\author{
(c) 2011. В. Л. ГЕйнц, В. В. Филиппов
}

В [1] Э. Майкл доказал следующую фундаментальную теорему.

Теорема 1. Пусть $F: X \rightarrow L-$ полунепрерьвное снизу многозначное отображсние паракомпакта $X$ в банахово пространство $L$, значения которого яв- 
ляются (непустыми) выпуклыми замкнутыми множествами. Тогда оно обладает непрерьвной селекиией.

В [2] сформулирован следующий вопрос. Пусть $Y-$ выпуклое $G_{\delta}$-nодмножество банахова пространства L. Будет ли всякое полунепреръвное снизу многозначное отображсение $F: X \rightarrow Y$ паракомпакта $X$ с выпуклыми замкнутыми в $Y$ значениями обладать непрерьвной селекиией?

В [3], [4] построением соответствующего примера был дан отрицательный ответ на этот вопрос.

В [5] доказана следующая теорема.

Пусть $Y$ - выпуклое $G_{\delta}$-подмножество банахова пространства E, удовлетворяющее условию (M): если $K \subset Y$ - компакт, то его замкнутая (в $Y)$ выпуклая оболочка - тоже компакт. Тогда всякое полунепрерывное снизу многозначное отображение паракомпакта $X$ в $Y$ с выпуклыми замкнутыми в $Y$ значениями обладает непрерьвной селекцией.

При обсуждении результатов работ [3]-[5] Майкл сформулировал вопрос: существенно ли в предположении теоремы условие, что $Y$ есть $G_{\delta}$-подмножество?

В этой заметке мы дадим положительный ответ на последний вопрос. Построение соответствующего примера будет опираться на исследование топологических свойств выпуклых оболочек некоторых компактных множеств вероятностных мер на отрезке.

1. Пространство $\boldsymbol{P}_{\boldsymbol{A}}$. Под пространством мер на отрезке $[0,1]$ мы будем понимать $C^{\prime}[0,1]$ - сопряженное к пространству непрерывных на отрезке $[0,1]$ действительных функций; всюду далее $C^{\prime}[0,1]$ рассматриваем с $*$-слабой топологией. Мера $\mu$ называется вероятностной, если $\mu(\varphi) \geqslant 0$ для любой функции $\varphi \in C[0,1], 0 \leqslant \varphi$, и $\mu(1)=1$. Обозначим через $P$ пространство вероятностных мер (с индуцированной из $C^{\prime}[0,1]$ топологией). Существует вложение пространства $P$ в гильбертов куб с сохранением выпуклой структуры; см., например, [3], [4]. Поэтому $P$ можно рассматривать как выпуклое подмножество гильбертова пространства $l_{2}($ над $\mathbb{R})$.

Пусть $\mu \in P$ и $x \in[0,1]$. Введем число $\tilde{\mu}_{x}=\inf \{\mu(\varphi) \mid \varphi:[0,1] \rightarrow[0,1], \varphi \in$ $C[0,1], \varphi(x)=1\}$ (мера точки $x$ относительно $\mu$ ). Положим $S_{\mu}:=\{x \mid x \in$ $\left.[0,1], \tilde{\mu}_{x}>0\right\}$. Следующие два утверждения очевидны.

Лемма 1. Для любой меръ $\mu \in P$ множество $S_{\mu}$ не более чем счетно.

Лемма 2. Для любой меры $\mu \in P$ въполняется неравенство $\sum_{x \in S_{\mu}} \tilde{\mu}_{x} \leqslant 1$.

Пусть $\delta_{x}$ - мера, сосредоточенная в точке $x$, т. е. $\delta_{x}(\varphi)=\varphi(x)$ для всякой $\varphi \in C[0,1]$. Пусть также $A=\left\{x_{i}: i=1,2, \ldots\right\}$ - счетное подмножество отрезка $[0,1]$ (если $i \neq j$, то $x_{i} \neq x_{j}$ ). Положим $P_{A}=\left\{\sum_{x \in A} \lambda_{x} \delta_{x} \mid \lambda_{x} \geqslant 0, \sum_{x \in A} \lambda_{x}=1\right\}$.

Ясно, что $P_{A} \subseteq P$ и множество $P_{A}$ выпукло.

Лемма 3. Во введенных выше обозначениях вероятностная мера $\mu$ принадлежит множеству $P_{A}$ тогда и только тогда, когда $S_{\mu} \subseteq A u \sum_{x \in S_{\mu}} \tilde{\mu}_{x}=1$.

Доказательство. I. Пусть $\mu \in P_{A}, \mu=\sum_{x \in A} \lambda_{x} \delta_{x}$. Тогда если $x \in[0,1] \backslash A$, то $\tilde{\mu}(x)=0$. Поэтому $S_{\mu} \subseteq A$. Если же $x \in S_{\mu}$, то $\tilde{\mu}(x)=\lambda_{x}$, так что $\sum_{x \in S_{\mu}} \tilde{\mu}_{x}=1$.

II. Обратно, пусть $S_{\mu} \subseteq A$ и $\sum_{x \in S_{\mu}} \tilde{\mu}_{x}=1$. Мера $\nu=\sum_{x \in S_{\mu}} \tilde{\mu}_{x} \delta_{x}$ принадлежит $P_{A}$. Наша цель - доказать, что $\mu=\nu$. Занумеруем точки, принадлежащие 
$S_{\mu}: S_{\mu}=\left\{x_{i}: i=1,2, \ldots\right\}$. Пусть $f:[0,1] \rightarrow[0,1]-$ непрерывная функция. Докажем, что $|\mu(f)-\nu(f)| \leqslant \varepsilon$ для любого $\varepsilon \in(0,1)$.

Возьмем такое $N \in \mathbb{N}$, что $\tilde{\mu}_{x_{N+1}}+\tilde{\mu}_{x_{N+2}}+\cdots<\varepsilon / 9$. Найдутся непересекающиеся интервалы $O x_{i} \ni x_{i}, i=1, \ldots, N$, и непрерывные функции $\varphi_{i}:[0,1] \rightarrow$ $[0,1]$, такие, что $\operatorname{supp}\left(\varphi_{i}\right) \subset O x_{i}, \tilde{\mu}_{x_{i}} \leqslant \mu\left(\varphi_{i}\right), \nu\left(\varphi_{i}\right) \leqslant \tilde{\mu}_{x_{i}}+\varepsilon /(9 N)$, а при $t \in O x_{i}$ имеют место неравенства $f\left(x_{i}\right)-\varepsilon /(9 N) \leqslant f(t) \leqslant f\left(x_{i}\right)+\varepsilon /(9 N)$.

Пусть $\Phi=\varphi_{1}+\cdots+\varphi_{N}$. Тогда

$$
\begin{gathered}
\tilde{\mu}_{x_{1}}+\cdots+\tilde{\mu}_{x_{N}} \leqslant \mu(\Phi), \nu(\Phi) \leqslant \tilde{\mu}_{x_{1}}+\cdots+\tilde{\mu}_{x_{N}}+\varepsilon / 9, \\
1-\tilde{\mu}_{x_{1}}-\cdots-\tilde{\mu}_{x_{N}}-\varepsilon / 9 \leqslant \mu(1-\Phi), \nu(1-\Phi) \leqslant 1-\tilde{\mu}_{x_{1}}-\cdots-\tilde{\mu}_{x_{N}} .
\end{gathered}
$$

При любом $i=1, \ldots, N$

$$
\begin{aligned}
\tilde{\mu}_{x_{i}} f\left(x_{i}\right)-\frac{\varepsilon}{9 N} & \leqslant \tilde{\mu}_{x_{i}}\left(f\left(x_{i}\right)-\frac{\varepsilon}{9 N}\right) \leqslant \mu\left(f \varphi_{i}\right), \nu\left(f \varphi_{i}\right) \\
& \leqslant\left(\tilde{\mu}_{x_{i}}+\frac{\varepsilon}{9 N}\right)\left(f\left(x_{i}\right)+\frac{\varepsilon}{9 N}\right) \leqslant \tilde{\mu}_{x_{i}} f\left(x_{i}\right)+\frac{\varepsilon}{3 N}
\end{aligned}
$$

поэтому

$$
\begin{aligned}
\tilde{\mu}_{x_{1}} f\left(x_{1}\right)+\cdots+\tilde{\mu}_{x_{N}} f\left(x_{N}\right)-\frac{\varepsilon}{9} & \leqslant \mu(f \Phi), \nu(f \Phi) \\
& \leqslant \tilde{\mu}_{x_{1}} f\left(x_{1}\right)+\cdots+\tilde{\mu}_{x_{N}} f\left(x_{N}\right)+\frac{\varepsilon}{3}
\end{aligned}
$$

и

$$
|\mu(f \Phi)-\nu(f \Phi)| \leqslant \frac{\varepsilon}{9}+\frac{\varepsilon}{3}=\frac{4 \varepsilon}{9}<\frac{\varepsilon}{2} .
$$

Кроме того,

$$
\begin{aligned}
& \mu(f-f \Phi) \leqslant \mu(1-\Phi) \leqslant 1-\tilde{\mu}_{x_{1}}-\cdots-\tilde{\mu}_{x_{N}}=\tilde{\mu}_{x_{N+1}}+\tilde{\mu}_{x_{N+2}}+\cdots<\varepsilon / 9 \\
& \nu(f-f \Phi) \leqslant \nu(1-\Phi) \leqslant 1-\tilde{\mu}_{x_{1}}-\cdots-\tilde{\mu}_{x_{N}}=\tilde{\mu}_{x_{N+1}}+\tilde{\mu}_{x_{N+2}}+\cdots<\varepsilon / 9 .
\end{aligned}
$$

Таким образом,

$$
\begin{aligned}
|\mu(f)-\nu(f)| & =|\mu(f \Phi)-\nu(f \Phi)+\mu(f-f \Phi)-\nu(f-f \Phi)| \\
& \leqslant|\mu(f \Phi)-\nu(f \Phi)|+|\mu(f-f \Phi)|+|\nu(f-f \Phi)| \leqslant \frac{\varepsilon}{2}+\frac{\varepsilon}{9}+\frac{\varepsilon}{9}<\varepsilon
\end{aligned}
$$

что и дает требуемое.

III. Случай произвольной непрерывной функции $f$ сводится к случаю II. В самом деле, пусть $|f| \leqslant M, M>0$. Функция $f_{1}=1 / 2+f /(2 M)$ удовлетворяет условию из II. Поэтому $1 / 2+\mu(f) /(2 M)=\mu\left(f_{1}\right)=\nu\left(f_{1}\right)=1 / 2+\nu(f) /(2 M)$, откуда и следует, что $\mu=\nu$. Лемма доказана.

Напомним, что $P$ мы рассматриваем с топологией $\tau$, индуцированной $*$-слабой топологией в $C^{\prime}[0,1]$. Поскольку $P$ с помощью некоторого топологически инъективного отображения, сохраняющего выпуклую структуру, отождествляется с выпуклым компактным подмножеством пространства $l_{2}$, то условие (M) для выпуклого подмножества $P_{A}$ гильбертова пространства $l_{2}$ можно сформулировать так: если множество $K \subseteq P_{A} \tau$-компактно, то и его $\tau$-замкнутая (в $\left.P_{A}\right)$ выпуклая оболочка $\tau$-компактна.

Теорема 2. Множество $P_{A}$ удовлетворяет условию (M). 
Доказательство. Установим следующий чуть более сильный факт. Пусть во введенных выше обозначениях $K \subseteq P_{A}, K-$ компакт. Тогда замкнутая выпуклая оболочка $c c(K)=c c_{P}(K)$ компакта $K$ в пространстве $P$ (компактна и) лежит в $P_{A}$.

Предположим противное: пусть существует мера $m \in c c(K) \backslash P_{A}$. Пусть $A=$ $\left\{x_{1}, x_{2}, \ldots\right\}$. Тогда, согласно лемме $3, \sum_{i=1}^{\infty} \widetilde{m}_{x_{i}}=1-\varepsilon, \varepsilon>0$. Выберем такие непрерывные $f_{i}:[0,1] \rightarrow[0,1]$, что $m\left(f_{i}\right)<\widetilde{m}_{x_{i}}+\varepsilon /\left(2 \cdot 2^{i}\right), f_{i}\left(x_{i}\right)=1$. Тогда $\sum_{i=1}^{\infty} m\left(f_{i}\right)<1-\varepsilon / 2$. Положим $F_{i}=\max \left\{f_{1}, \ldots, f_{i}\right\}$ для $i \in \mathbb{N}$. Мы имеем неубывающую последовательность функций $F_{n}$, причем $m\left(F_{n}\right) \leqslant \sum_{i=1}^{\infty} m\left(f_{i}\right)<$ $1-\varepsilon / 2$.

Рассмотрим теперь последовательность окрестностей $O_{i}(m)=\{\mu \in P$ : $\left.\mu\left(F_{i}\right)<1-\varepsilon / 2\right\}$ меры $m$. Поскольку $\left\{F_{n}\right\}_{n=1}^{\infty}$ не убывает, то $\left\{O_{i}(m)\right\}_{i=1}^{\infty}$ не возрастает. Предположим, что $O_{i}(m) \cap K=\varnothing$. Тогда $K \subseteq P \backslash O_{i}(m)$, но $O_{i}(m)$ и ее дополнение - множества выпуклые; следовательно, поскольку $O_{i}(m)$ открыто, $c c(K) \subseteq P \backslash O_{i}(m)$, что невозможно, так как $m \in c c(K) \cap O_{i}(m)$. Значит, $O_{i}(m) \cap K \neq \varnothing$ для любого $i \in \mathbb{N}$.

Имеем невозрастающую последовательность компактов $K \cap\left[O_{i}(m)\right] \neq \varnothing$. Ее пересечение $K_{0}$ непусто. Пусть $\eta \in K_{0}$. Тогда $\eta \in\left[O_{k}(m)\right]$ для $k=1,2, \ldots$; поэтому $\eta\left(F_{k}\right) \leqslant 1-\varepsilon / 2$ и $\sum_{i=1}^{k} \tilde{\eta}_{x_{i}} \leqslant \eta\left(F_{k}\right) \leqslant 1-\varepsilon / 2$. Переходя к пределу, получаем $\sum_{i=1}^{\infty} \tilde{\eta}_{x_{i}} \leqslant 1-\varepsilon / 2<1$, а значит, $\eta \notin P_{A}$. Полученное противоречие и компактность пространства $P$ завершают доказательство утверждения.

2. Первый пример. Пусть $M_{1}=\left\{x_{i}: i=1,2, \ldots\right\}$ - множество рациональных точек отрезка $[0,1], M_{2}=[0,1] \backslash M_{1}, M_{3}=\left\{y_{i}: y_{i}=x_{i} \sqrt{3}, x_{i} \sqrt{3} \in\right.$ $(0,1), i=1,2, \ldots\}$ (если $i \neq j$, то $x_{i} \neq x_{j}$ и $\left.y_{i} \neq y_{j}\right)$.

Обозначим через $Y$ подмножество $\left(M_{1} \times P_{M_{1}}\right) \cup\left(M_{2} \times P_{M_{3}}\right)$ произведения $[0,1] \times P$. Многозначное отображение $G:[0,1] \rightarrow P$ определим так:

$$
G(x)= \begin{cases}P_{M_{1}} & \text { для } x \in M_{1}, \\ P_{M_{3}} & \text { для } x \in M_{2} .\end{cases}
$$

Многозначное отображение $F$ отрезка $[0,1]$ в $Y$ зададим как диагональное произведение (см. [7]) тождественного отображения $J:[0,1] \rightarrow[0,1]$ и отображения $G$, т. е. $F(x)=\{x\} \times G(x)$. Нетрудно проверить, что для полунепрерывности снизу диагонального произведения многозначных отображений $\chi$ и $\psi$ пространства $X$ в пространства $Y$ и $Z$ соответственно достаточно полунепрерывности снизу отображений $\chi$ и $\psi$.

Утверждение 1. Отображение $F$ полунепрерывно снизу.

Доказательство. Из плотности множеств $M_{1}$ и $M_{3}$ в $[0,1]$, плотности множества конечных выпуклых комбинаций мер Дирака в $P$ и непрерывности отображения $x \mapsto \delta_{x}$ следует, что $P_{M_{1}}$ и $P_{M_{3}}$ плотны в $P$. Отсюда вытекает полунепрерывность снизу отображения $G$. Кроме того, $J$ непрерывно, а следовательно, $F$ полунепрерывно снизу.

Утверждение 2. Отображение $F$ не допускает непрерывной селекции.

Доказательство будет напоминать соответствующее рассуждение в статье [1]. Предположим противное. Тогда у отображения $G$ существует непрерывная 
однозначная селекция $f:[0,1] \rightarrow P$. Найдется последовательность непрерывных на $[0,1]$ функций $\varphi_{i}$, такая, что $\varphi_{i}\left(x_{k}\right)=0$ при $k \leqslant i$ и $\varphi_{i}\left(y_{k}\right)=1$ при $k \leqslant i$. Ввиду непрерывности селекции $f$ для каждого $x_{k}$ найдется окрестность $U_{k}$, такая, что для любых двух точек $s_{1}, s_{2} \in U_{k}$ выполнено неравенство

$$
\left|f\left(s_{1}\right)\left(\varphi_{k}\right)-f\left(s_{2}\right)\left(\varphi_{k}\right)\right|<1 / 2 .
$$

Положим $I_{0}=[0,1]$. Выберем произвольную рациональную точку $x_{n_{1}}$ внутри $I_{0}$, затем в ее окрестности $U_{n_{1}} \cap \operatorname{int}\left(I_{0}\right)$ выберем отрезок $I_{1}$ длины $d_{1}<1 / 2$, содержащий внутри себя $x_{n_{1}}$ и не содержащий $x_{m}$ при $m<n_{1}$. Затем внутри $I_{1}$ выберем $x_{n_{2}}, n_{2}>n_{1}$, и отрезок $I_{2}$ длины $d_{2}<1 / 4$, причем $I_{2} \subset U_{n_{2}} \cap \operatorname{int}\left(I_{1}\right)$, $x_{n_{2}} \in \operatorname{int}\left(I_{2}\right), x_{m} \notin I_{2}$ при $m<n_{2}$, и т.д. Тогда $\bigcap_{n=1}^{\infty} I_{n}=\{t\}, t \in M_{2}$. Значит, $f(t)=\sum_{i=1}^{\infty} \lambda_{i} \delta_{y_{i}}$. Существует $k \in \mathbb{N}$, такое, что $t \in U_{k}$ и $\sum_{i=k+1}^{\infty} \lambda_{i}<1 / 4$. Тогда $f(t)\left(\varphi_{k}\right)=\sum_{i=1}^{\infty} \lambda_{i} \varphi_{k}\left(y_{i}\right)>(1-1 / 4)$, a $f\left(x_{k}\right)\left(\varphi_{k}\right)=\sum_{i=1}^{\infty} \lambda_{i} \varphi_{k}\left(x_{i}\right)<1 / 4$. Разность между $f(t)\left(\varphi_{k}\right)$ и $f\left(x_{k}\right)\left(\varphi_{k}\right)$ оказалась больше $1 / 2$, что противоречит условию $(*)$. Утверждение доказано.

3. Второй пример. В примере разд. 2 условию (M) удовлетворяют образы точек, но пространство $Y$ в целом этому условию не удовлетворяет, поскольку $Y$ не выпукло. В этом разделе мы немного усложним построение и добьемся выполнения условия (M) для пространства $Y^{\prime}$ в целом. Фактически мы вложим построенный в разд. 2 пример в пространство, удовлетворяющее этому условию.

Сохраним введенные ранее обозначения. Пусть $D=\left\{(\xi, \eta) \mid \xi^{2}+\eta^{2} \leqslant 1\right\}$, $S=\left\{(\xi, \eta) \mid \xi^{2}+\eta^{2}=1\right\}, \alpha(x)=(\cos x, \sin x), M_{1}^{\prime}=\left\{\alpha(x) \mid x \in M_{1}\right\}, M_{2}^{\prime}=$ $\left\{\alpha(x) \mid x \in M_{2}\right\}$. Положим $Y^{\prime}=((D \backslash S) \times P) \cup\left(M_{1}^{\prime} \times P_{M_{1}}\right) \cup\left(M_{2}^{\prime} \times P_{M_{3}}\right) \subset D \times P$. Множество $Y^{\prime}$ рассматриваем как подмножество банахова пространства $E^{\prime}=$ $\mathbb{R}^{2} \times l_{2}$. Оно выпукло ввиду строгой выпуклости $D$. Многозначное отображение $F^{\prime}:[0,1] \rightarrow Y^{\prime}$ зададим так: $F^{\prime}(x)=\{\alpha(x)\} \times G(x)$.

Лемма 5. Если $A$ и $B$ - выпуклые компакты в банаховом пространстве $E$, mo $c c(A \cup B)=\bigcup\{[x, y] \mid x \in A, y \in B\}$.

Доказательство. Множество $\bigcup\{[x, y] \mid x \in A, y \in B\}$ - это образ компакта $A \times B \times[0,1]$ при непрерывном отображении $H:(a, b, t) \mapsto t a+(1-t) b$; поэтому оно компактно. Его выпуклость очевидна.

Утверждение 1. Множество $Y^{\prime}$ удовлетворяет условию (M).

Доказательство. Предположим, что $K$ - компактное подмножество множества $Y^{\prime}$. Требуется доказать, что его замкнутая выпуклая оболочка в $Y^{\prime}-$ компакт. Вместо $c c_{D \times P}(K)$ пишем для краткости $c c(K)$. Ясно, что $c c(K)-$ компакт.

Наша цель будет достигнута, если мы покажем, что $c c(K) \subseteq Y^{\prime}$. Допустим противное. Тогда найдется точка $p=\left(p_{1}, p_{2}\right) \in c c(K) \backslash Y^{\prime}$, причем $p_{1} \in S$.

Пусть $Q=\left\{q \mid\left(p_{1}, q\right) \in K\right\}$. Если $p_{2} \in c c_{P}(Q)$, то по теореме $2\left(p_{1}, p_{2}\right) \in Y^{\prime}$, поскольку $Q$ - компактное подмножество в $P_{M_{1}}$ или в $P_{M_{2}}$. Предположим теперь, что $p_{2} \notin c c_{P}(Q)$. По теореме Хана-Банаха (см. [6]) существует непрерывный линейный функционал $f$ на пространстве $l_{2}$ и число $c$, такие, что $\sup \left\{f(x) \mid x \in c c_{P}(Q)\right\}<c<f\left(p_{2}\right)$.

Пусть $K_{1}=\{(s, t) \in K \mid f(t) \geqslant c\}$ и $K_{2}=\{(s, t) \in K \mid f(t) \leqslant c\}$. Тогда $K_{1}$ - компакт, проекция $\widetilde{K}_{1}$ которого на $D$ не содержит $p_{1}$. Но тогда, поскольку $p_{1} \in S$, число $c_{1}=\sup \left\{\left(s, p_{1}\right) \mid s \in \widetilde{K}_{1}\right\}$ (где $()-$, скалярное произведение) 
строго меньше 1. Множество $\bar{K}_{1}=\left\{(s, t) \mid s \in D, t \in P,\left(p_{1}, s\right) \leqslant c_{1}\right\}$ выпукло, компактно и содержит $K_{1}$; поэтому $c c\left(K_{1}\right) \subseteq \bar{K}_{1}$.

Так как $p \in c c(K)$ и $K=K_{1} \cup K_{2}$, а $c c\left(K_{1}\right)$ и $c c\left(K_{2}\right)$ - выпуклые компакты в $D \times P$, то точка $p$ лежит на отрезке, соединяющем какие-то две точки $x \in c c\left(K_{1}\right)$ и $y \in c c\left(K_{2}\right)$. При этом $p \neq x$ и $p \neq y$, поскольку $c<f\left(p_{2}\right)$. Но это возможно, только если $p_{1} \in D \backslash S$. Противоречие.

Из непрерывности отображения $x \mapsto \alpha(x)$ и утверждений примера 1 вытекают следующие факты.

Утверждение 2. Отображсние $F^{\prime}$ полунепрерьвно снизу.

Утверждение 3. Отображение $F^{\prime}$ не имеет непрерывной селекиии.

Из теоремы Майкла и Намиоки и построенного примера следует, что $Y^{\prime}$ не является $G_{\delta}$-подмножеством банахова пространства $\mathbb{R}^{2} \times l_{2}$.

\title{
ЛИТЕРАТУРА
}

[1] E. A. Michael, Ann. of Math., 63 (1956), 361-382. [2] E. A. Michael, in: Open Problems in Topology, North Holland, Amsterdam, 1990, 271-278. [3] V. V. Filippov, Comment. Math. Univ. Carolinae, 45:4 (2004), 735-737. [4] В. В. Филиппов, Матем. заметки, 78:4 (2005), 638-640. [5] I. Namioka, E. A. Michael, Topology Appl., 155:8 (2008), 858-860. [6] У. Рудин, Функционалъный анализ, Мир, М., 1975. [7] В. В. Федорчук, В. В. Филиппов, Общая топология. Основные конструкции, Изд-во МГУ, М., 1988.

Московский государственный университет e-mail: mir14mai@inbox.ru

Московский государственный университет e-mail:vvfil@mech.math.msu.su
Поступило в редакцию 31 июля 2009 г.

\section{УДК $517.588+512.548 .2$}

\section{Функция Мёбиуса на абелевых полугруппах}

\author{
(c) 2011. Е. А. Горин
}

1. Формулы обращения были открыты Мёбиусом в 30-е годы XIX века. Частные случаи встречаются у Гаусса, однако в общем объеме Гаусс их, вероятно, не знал, иначе Мёбиус, который учился у Гаусса (наблюдательной астрономии), не мог на него не сослаться. В середине века они были переоткрыты, в частности, П. Л. Чебышёвым и Б. Риманом (оба ни на кого не ссылаются).

В XX веке были обнаружены многочисленные обобщения, среди которых одним из наиболее удачных является найденное Ротой, который широко применял полученные им формулы к проблемам комбинаторики и геометрии. Теория Роты кратко описана в [1]. В этой теории исходным объектом служит локально конечное частично упорядоченное множество $X$. Роль $\zeta$-функции играет такая матрица $\rho$, что $\rho(x, y)$ равно 1 при $x \leqslant y$ и 0 в остальных случаях. Обратная к ней матрица играет роль функции Мёбиуса. Когда в качестве $X$ берется натуральный ряд с порядком $a \mid b$ (делимость нацело), формулы Роты согласуются 